Volume 19, No 4 International Journal of Radiation Research, October 2021

\title{
Liangxue Guyuan decoction alleviates radiation enteritis through PI3K/AKT pathway in rats
}

\author{
Y.G. Wang ${ }^{\#}$ H.C. Xu ${ }^{\#}$, J. Feng, C.Y. Xu, Y.Q. Dou ${ }^{*}$ \\ Senior Department of Traditional Chinese Medicine, Chinese People's Liberation Army General Hospital, Beijing \\ 100853, P. R. China
}

\begin{abstract}
Background: Liangxue Guyuan decoction (LGD) has been used to alleviate symptoms of radiation enteritis (RE) in the clinic. This study aimed to investigate the effects and anti-apoptosis and proliferative mechanisms of LGD in rats with RE. Materials and Methods: A total of 115 male Sprague Dawley rats were used. The control group consisted of 15 rats, and the remaining 100 rats were irradiated with ${ }^{60} \mathrm{Co} \gamma$ rays at a dose of $11 \mathrm{~Gy}$ and randomly divided into four groups of 25 each: the model group, the dexamethasone (DXM) group, the low-dose LGD (LGDI) group, and the highdose LGD (LGDh) group. The treatment was administered intragastrically for 7 days, and the number of rats that died was recorded daily. On days 3 and 7 after irradiation, five rats were randomly chosen and sacrificed from each group, and ileum samples were taken for pathological observation, immunofluorescence staining, western blotting, and PCR experiments. Results: Compared with the model group, the mean survival time, villus length $(\mathrm{V})$, and the ratio of $\mathrm{V}$ to crypt depth $\mathrm{C}(\mathrm{V} / \mathrm{C})$, the number of Ki67positive cells was increased, and the number of TUNEL-positive cells was reduced in the LGDh group. In addition, LGDh showed some effects on upregulating p-PI3K, p-AKT, and $\mathrm{Bcl}-2$ and downregulating caspase- 3 and Bax. The effect of LGD was better than that of DXM. Conclusions: LGDh prolonged the survival time of irradiated rats, inhibited intestinal epithelial cell apoptosis, and promoted intestinal repair. This mechanism may be related to the regulation of the PI3K/AKT pathway.
\end{abstract}

Keywords: Liangxue Guyuan decoction, radiation enteritis, PI3K/AKT pathway.

\section{INTRODUCTION}

Radiotherapy is an effective way to treat cancer, but it has side effects. Radiation enteritis (RE) is a common complication of pelvic cancer radiotherapy, which severely hinders the treatment process. After exposure to ionizing radiation, intestinal crypt cell apoptosis and intestinal epithelial cell regeneration are blocked, leading to RE, which can cause bacteremia, toxemia, and even death (1-2). Therefore, controlling the development of RE by inhibiting cell apoptosis and promoting proliferation is a key focus of current research. However, the treatment of RE is challenging, and there is a lack of effective methods. Epidermal growth factor (3), mesenchymal stem cells (4), and growth hormones (5) have certain clinical therapeutic effects, but these treatments have drawbacks, such as limited scope of action, side effects, and lack of clinical practice (6).

In traditional Chinese medicine, radiation is viewed a as mix of heat and toxicity, and the body must be detoxified and cooled during the course of treatment. After several years of clinical exploration and application, we found that Liangxue Guyuan decoction (LGD) can relieve abdominal pain, diarrhea, and hematochezia in RE patients and improve their quality of life. In addition, studies have shown that the constituents of LGD can inhibit apoptosis and promote intestinal repair. For example, the acidic polysaccharide of Panax ginseng regulates the mitochondria/caspase- 
dependent apoptotic pathway in radiation-induced damage to the jejunum (7). Cortex Moutan regulates the expression of malondialdehyde, superoxide dismutase, B-cell lymphoma-2 (Bcl-2), and Bcl-2-associated X protein (Bax) and exerts anti-oxidant and anti-apoptotic effects (8). Radix Rehmanniae increases the viability and proliferative capacity of the cells and alleviates $\mathrm{H}_{2} \mathrm{O}_{2}$-induced apoptosis of human adipose-derived mesenchymal stem cells (9). Radix Paeoniae Rubra inhibits cardiomyocyte apoptosis and protects against myocardial ischemic injury by activating the PI3K/AKT/mTOR signaling pathway (10). Extracts of Cornu Bubali have anti-oxidant effects (11).

As a well-established anti-apoptotic signaling pathway, the phosphatidylinositol- 3-kinase $(\mathrm{PI} 3 \mathrm{~K}) /$ protein kinase $\mathrm{B}$ (AKT) pathway is closely related to cell apoptosis and proliferation (12). Studies have shown that radiation can activate PI3K, induce downstream phosphoinositol-dependent kinase 1 to activate AKT, which not only participates in cell proliferation but also activates Bcl-2 to play an anti-apoptotic role, reduce the production of Bax and caspase- 3 to inhibit apoptosis, and promote apoptosis. Therefore, in this study, we examined the effect of LGD on RE rats and explored its anti -apoptosis and proliferative mechanism by investigating its effects on the PI3K/AKT pathway.

$\mathrm{RE}$ is a problem faced by patients on a global scale. Many clinical practices and clinical studies have shown that traditional Chinese medicine has a good effect on RE. Moreover, LGD is a common prescription in our clinical practice, which can relieve pain and improve the quality of life of many patients. Therefore, this project aimed to provide new ideas and methods for the treatment of this disease.

\section{MATERIALS AND METHODS}

\section{Animals}

Male Sprague Dawley rats $(235 \pm 20$ g) were purchased from Vital River Laboratory Animal Technology Co., Ltd. (Beijing, China). The rats were kept in the Laboratory Animal Center of the Academy of Military Medical Sciences (Beijing, China) under laboratory conditions of $25 \pm 2{ }^{\circ} \mathrm{C}$, relative humidity of $55 \pm 2 \%$, and a $12 \mathrm{~h}$ dark/light cycle. The study was approved by the experimental animal ethics committee of Chinese People's Liberation Army General Hospital.

\section{Drugs}

The constituents of LGD are shown in table 1. All the herbs were purchased from Luye Pharmaceutical Co., Ltd. (Beijing, China). The herbs were soaked in 10-times distilled water for $30 \mathrm{~min}$ and boiled for $60 \mathrm{~min}$. Four-times distilled water was added to the residue and decocted twice for $30 \mathrm{~min}$. All collected supernatants were filtered through eight layers of gauze and concentrated with a rotary evaporator. Finally, the extract was sterilized under high pressure and stored at $-20^{\circ} \mathrm{C}$. This preparation method follows the traditional method and is also the same as that used for clinical preparation.

Ultrahigh-Performance Liquid Chromatography Electrospray Ionization Q-Orbitrap Mass Spectrometry method was used to determine its main components. The daily dose of LGD in humans is $100 \mathrm{~g} / 60 \mathrm{~kg}$. According to the formula $d_{\text {rat }}=d_{\text {human }} \times 0.7 / 0.11$, where $d_{\text {rat }}$ and $d_{\text {human }}$ are the rat and human doses, respectively, we decided to use 9.01 and $36.04 \mathrm{~g} / \mathrm{kg} /$ day as the low and high doses of LGD. Dexamethasone (H33020822, ZheJiang Xianju Pharmaceutical Co., Ltd.) was used as the positive control.

\section{Modeling, grouping, and drug administration}

After three days of acclimatization, 110 male Sprague Dawley rats were randomly divided into five groups: control $(n=10)$, model $(n=25)$, dexamethasone (DXM, $n=25$ ), low-dose LGD (LGDl, $n=25$ ), and high--dose LGD (LGDh, $n=25$ ). We irradiated all rats except the control group using a ${ }^{60} \mathrm{Co}$ source (Institute of Radiation Medicine and Radiation Protection Medicine, Academy of Military Medical Sciences, Beijing, China), and the total exposure dose was $11 \mathrm{~Gy}$. The control group and model group were given normal saline by intragastric administration

Int. J. Radiat. Res., Vol. 19 No. 4, October 2021 
once daily. The DXM group was given dexamethasone solution at dosage of $1.425 \mathrm{mg} /$ $\mathrm{kg}$.

\section{Sample collection}

On day 3 and 7 after irradiation, five rats of each group were anesthetized with ether and sacrificed. To examine the ileum histopathological changes and explore the underlying molecular biological mechanism, the ileum was removed. Half of the ileum was fixed in $10 \%$ paraformaldehyde for hematoxylin and eosin (H\&E) staining and immunohistochemistry, whereas the other half was kept in a cryotube for western blotting and RT-PCR.

\section{Survival time}

The number of rats that died and time of death were recorded daily.

\section{Histological examination}

Transversal slices of ileum were fixed in $4 \%$ buffered formaldehyde at $4{ }^{\circ} \mathrm{C}$ for $24 \mathrm{~h}$ and embedded in paraffin. The sections were stained with H\&E and observed by light microscopy (CX31, Olympus, Japan). The villus height (V), crypt depth $(C)$, and $V / C$ ratio were measured by a pathologist.

\section{TUNEL assay}

The sections were treated according to the manufacturer's protocols (11684817910, Roche, Germany). The sections were observed under a fluorescence microscope (Eclipse C1, Nikon, Japan) and the images were collected with a digital camera system (DS-U3, Nikon).

\section{Ki67 immunofluorescence staining}

The slices were dewaxed and rehydrated with citrate buffer. Then, the slices were boiled in $10 \mathrm{mM} / \mathrm{L}$ citric acid buffer and the antigens were extracted according to the standard procedure. After incubating with serum at room temperature for $1 \mathrm{~h}$, sections were incubated overnight with anti-Ki67 antibody (GB21303, Goodbio, China) at $4^{\circ} \mathrm{C}$, and then incubated with secondary antibody at $37^{\circ} \mathrm{C}$ for 30 minutes. The positive cells were detected by DAPI (G1012,
Goodbio, China). The positive cells were counted using fluorescence microscope (Nikon Eclipse C1, Japan).

\section{Western blotting}

Western blotting was used to detect the expression levels of Bax, Bcl-2, and caspase- 3 proteins. The tissues were first homogenized with RIPA lysates (G2002, Goodbio, China) and centrifuged at $12,000 \times \mathrm{g}$ and $4{ }^{\circ} \mathrm{C}$ for $10 \mathrm{~min}$, to obtain the supernatant. The BCA method was used to detect protein concentration, according to the manufacturer's instructions (G2026, Goodbio). The loading volume was measured according to the protein concentration. All protein samples were separated by SDS-PAGE and subsequently transferred onto polyvinylidene fluoride membranes. Then, the membranes were blocked with $5 \%$ skim milk and incubated with corresponding primary antibodies $\left(4{ }^{\circ} \mathrm{C}\right.$, overnight). After cleaning with Tris-buffered saline and TBST, secondary antibodies were added. The membrane was incubated for 30 min, ECLA and ECLB reagents (G2014, Goodbio) were mixed and were incubated with the membrane for $2 \mathrm{~min}$, and then the membrane was exposed in a dark box. Finally, the gray values of all samples were measured with software (alpha Ease FC, Alpha Innotech). Primary antibodies included p-PI3K (AF3241, 1:1000, Affinity, China), p-Akt (AF0908, 1:1000, Affinity, China), Bax (GB11007, 1:1000, Servicebio, China), caspase-3 (66470-2-lg, 1:1000, San Ying, China), Bcl-2 (ab59348, 1:1000, Abcam, UK), and ACTIN (GB12001, 1:1000, Servicebio, China).

\section{PCR}

The transcription levels of Bax, Bcl-2, and caspase- 3 were measured using PCR. Total RNA was extracted using TRIzol (G3013, Servicebio), and the synthesis of cDNA was performed using reverse transcriptase. Reverse transcriptase reactions were performed in a reaction mixture of $1 \mu \mathrm{L}$ Oligo (dT), $4 \mu \mathrm{L} 5 \times$ Reaction Buffer, $2 \mu \mathrm{L}$ $10 \mathrm{mM}$ dNTP Mix, $1 \mu \mathrm{L}$ RiboLock RNAase inhibitor $(20 \mathrm{U} / \mu \mathrm{L})$, and $1 \mu \mathrm{L}$ RevertAi M-MuLV $(200 \mathrm{U} / \mu \mathrm{L})$. The reaction mixtures were 
incubated first at $42{ }^{\circ} \mathrm{C}$ for $60 \mathrm{~min}$, and then at $70{ }^{\circ} \mathrm{C}$ for $5 \mathrm{~min}$. RT-PCR for each cDNA was performed in triplicate in $20 \mu \mathrm{L}$ of reaction mixture. Reactions were incubated with an RT-PCR system (StepOnePlus, Applied Biosystems, USA) at $95{ }^{\circ} \mathrm{C}$ for $10 \mathrm{~min}$, followed by 40 cycles of $95^{\circ} \mathrm{C}$ for $15 \mathrm{~s}$ and $60^{\circ} \mathrm{C}$. The level of gene expression was quantified using a standard curve, and we used the formula $2^{-\Delta \Delta C T}$ to calculate the relative expression levels of genes. In a parallel experiment, glyceraldehyde 3 -phosphate dehydrogenase (GAPDH) was used as an internal control. The primer sequences are shown in table 2 .

\section{Statistical analysis}

The results are presented as mean \pm standard deviation (SD). All statistical analyses were performed using SPSS 21.0 statistical software (SPSS, USA). One-way analysis of variance was used to examine the differences between groups. $\mathrm{P}<0.05$ was considered statistically significant.

Table 1. Constituents of LGD.

\begin{tabular}{|c|c|c|c|c|}
\hline Chinese name & English name & Part used & Origin /batch number & Amount(g) \\
\hline Sheng Shai Shen & Panax ginseng & Root & Hunan (China)/1705271 & 20 \\
\hline Shui Niu Jiao & Cornu Bubali & Horn & Jilin (China)/17022602 & 20 \\
\hline Dan Pi & Cortex Moutan & Root & Anhui (China)/17111701 & 15 \\
\hline Chi Shao & Radix Paeoniae Rubra & Root & Neimeng (China)/18081101 & 15 \\
\hline Sheng Di & Radix Rehmanniae & Root & He Nan (China)/18081001 & 15 \\
\hline
\end{tabular}

Table 2. Primer sequences.

\begin{tabular}{|c|c|c|}
\hline Primer & Sequence (5' to $\mathbf{3}^{\prime}$ ) & Length \\
\hline Caspase-3 & $\begin{array}{c}\text { FW: GAAAGCCGAAACTCTTCATCAT } \\
\text { RV: ATGCCATATCATCGTCAGTTCC }\end{array}$ & $98 \mathrm{bp}$ \\
\hline Bax & $\begin{array}{c}\text { FW: TGAACTGGACAACAACATGGAG } \\
\text { RV: AGCAAAGTAGAAAAGGGCAACC }\end{array}$ & $148 \mathrm{bp}$ \\
\hline Bcl-2 & $\begin{array}{r}\text { FW: TTGTGGCCTTCTTTGAGTTCG } \\
\text { RV: GCATCCCAGCCTCCGTTAT }\end{array}$ & $151 \mathrm{bp}$ \\
\hline GAPDH & $\begin{array}{c}\text { FW: CTGGAGAAACCTGCCAAGTATG } \\
\text { RV: GGTGGAAGAATGGGAGTTGCT }\end{array}$ & $138 \mathrm{bp}$ \\
\hline
\end{tabular}

\section{RESULTS}

\section{Effective components of LGD}

Data collected by high-resolution liquid and mass were collated by CD2.1 (Thermo Fisher, USA) for database retrieval and comparison. The fingerprint revealed eight main components of LGD: baicalin and albiflorin, the main component of Radix Paeoniae Rubra; catalpol and aucubin the main component of Rehmannia Glutinosa Libosch; ginsenoside $\mathrm{Rb} 1$, ginsenoside $\operatorname{Rg} 1$ and ginsenoside Rc the main component of Radix Ginseng; paeonilorin the main component of both Radix Paeoniae Rubra and Cortex Moutan.

\section{The survival time of irradiated rats}

The rats in the irradiated groups started to die as early as day 4 after irradiation (figure 2). Mean survival time values were 7.00 \pm 0 (control), $5.80 \pm 1.32$ (model), $6.53 \pm 0.92$ (DXM), $6.53 \pm 0.92$ (LGDl), and $6.67 \pm 0.82$ (LGDh) days. The mean survival time was longer in the LGDh group than in the model group $(\mathrm{P}<0.05)$. No significant difference was observed in the DXM and LGDl groups compared with the model group $(\mathrm{P}>0.05)$.

\section{Histopathological changes in the ileum}

The histological morphologies of the ilea on days 3 and 7 after radiation are shown in figure $3 \mathrm{~A}$. In the control group, the morphology of the ileum was intact. However, on day 3, short, thick villi, and partial villous epithelial necrosis were observed in the model group, whereas injury to the ileum was improved in the high-dose LGD 
(LGDh) group. The villus length and $V / C$ ratio of the irradiated groups were lower $(P<0.01)$ than those of the control group and higher than those of the model group $(P<0.05)$ (figure $3 \mathrm{~B}$ ). On day 7 , the length of the intestinal villi increased slightly, and the epithelial structure partially recovered in the irradiated groups. The villus lengths of the irradiated groups $(P<0.01)$ and the $V / C$ ratio of the model group and low-dose LGD (LGDl) group were lower $(P<0.05)$ (figure $3 \mathrm{C}$ ) than those of the control group.

\section{Effects of LGD on intestinal cell apoptosis on days 3 and 7}

The ilea in the irradiated groups $(P<0.01)$ on day 3 showed a significantly higher number of apoptotic cells than the control group. By contrast, the number of apoptotic cells was lower in the LGDl $(P<0.05)$ and LGDh $(\mathrm{P}<0.01)$ groups. On day 7 , the number of terminal deoxynucleotidyl transferase nick-end labeling (TUNEL)-positive cells in the model group $(P<0.01)$ was still higher than that in the control group. However, in the LGDh group $(P<0.05)$ alone, the number of TUNEL-positive cells decreased compared with that in the model group (figure 4).

\section{Effects of LGD on intestinal cell proliferation on days 3 and 7}

Ki67 immunofluorescence staining is shown in figure $5 \mathrm{~A}$ and $\mathrm{B}$. On days 3 and 7, the Ki67-positive cells in the irradiated groups decreased significantly $(P<0.01)$. On day 3 , the number of Ki67-positive cells in the LGDl and LGDh groups increased, compared with that in the model group $(P<0.01)$ (Figure $5 \mathrm{C}(\mathrm{a}))$. On day 7 , the number of Ki67-positive cells increased only in the LGDh group $(P<0.05)$ (figure 5 C (b)).

\section{Effects of LGD on the expression of PI3K/AKT pathway-related proteins \\ on day 3}

The protein levels of caspase- $3(\mathrm{P}<0.01)$ and Bax $(\mathrm{P}<0.05)$ were higher and the protein levels of Bcl-2 $(\mathrm{P}<0.05)$ were lower in the model group than in the control group. Compared with the model group, in the LGDh group, the p-PI3K level was high $(P<0.05)$ and the caspase-3 level was low $(P<0.05)$. The p-AKT and Bcl-2 levels in the LGDl $(P<0.05, P<0.05)$ and LGDh $(P<0.05$, $P<0.01$ ) groups were elevated (figure 6).

\section{Effects of LGD on the expression of PI3K/AKT pathway-related proteins on day 7}

Compared with the control group, in each treatment group, the p-AKT level had improved $(P<0.01$ or $P<0.05)$, and the levels of $\mathrm{p}-\mathrm{PI} 3 \mathrm{~K}$ $(P<0.05)$ were lower and of caspase-3 $(P<0.01)$ and Bax $(P<0.01)$ were higher in the model group than in the control group. P-PI3K level in the LGDh group $(P<0.01)$, p-AKT level in the LGDl $(P<0.05)$ and LGDh $(P<0.05)$ groups, and Bcl-2 level in each treatment group (all $P<0.05$ ) were higher and Bax expression in the LGDh group was lower $(P<0.05)$ than those in the model group (figure 7 ).

Effects of LGD on the mRNA expression of PI3K/AKT pathway-related factors on days 3 and 7

On day 3 , the mRNA levels of caspase-3 $(P<0.05)$ and Bax $(P<0.01)$ were higher and Bcl-2 level $(P<0.01)$ was lower in the model group than in the control group. However, in the LGDh group, the caspase- 3 mRNA expression $(P<0.05)$ decreased and Bcl-2 mRNA expression $(P<0.05)$ increased compared with that in the model group. On day 7, Bax mRNA level $(P<0.01)$ was still higher in the model group than in the control group. By contrast, Bax mRNA expression was lower in the LGDl and LGDh groups than in the model group $(P<0.05$ and $P<0.01$, respectively) (figure 8 ).

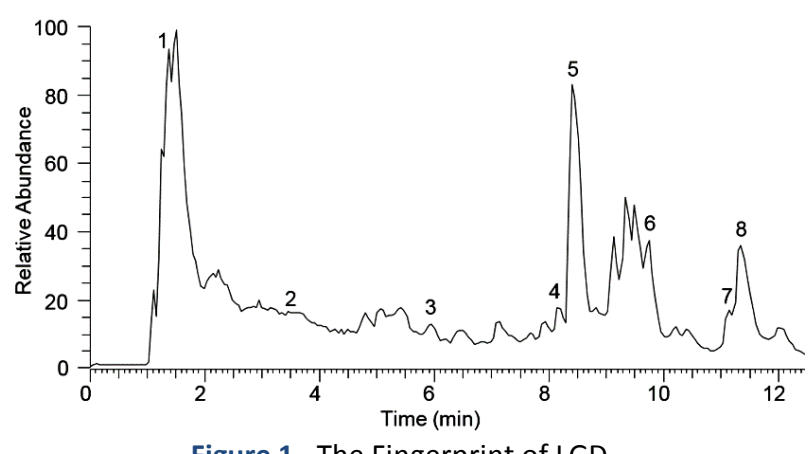

Figure 1. The Fingerprint of LGD.

Notes: 1: baicalin; 2: catalpol; 3: aucubin; 4: albiflorin; 5: paeoniflorin; 6: ginsenoside Rg1; 7: ginsenoside Rb1; 8: ginsenoside Rc. 


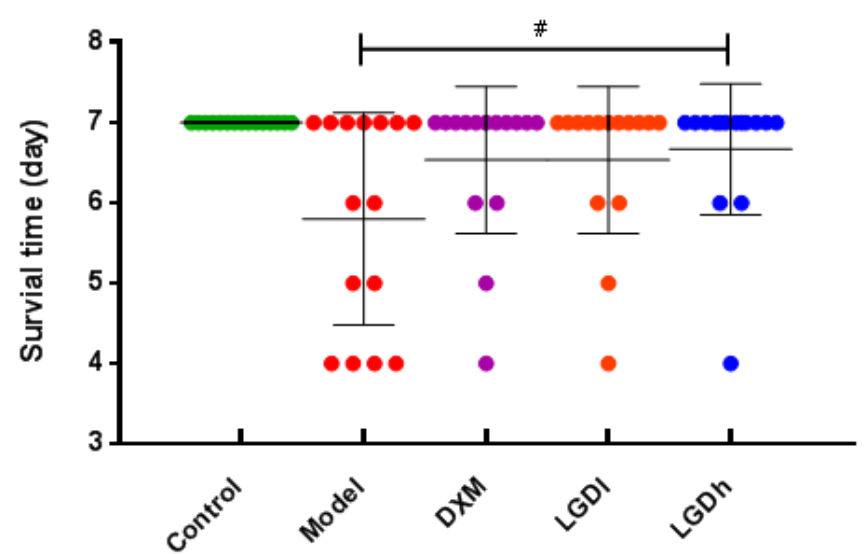

Figure 2. Effects of LGD on survival time of rats. \#P $<0.05$ versus model.

A
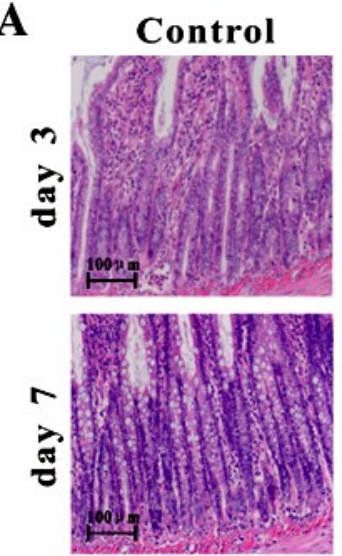

Model
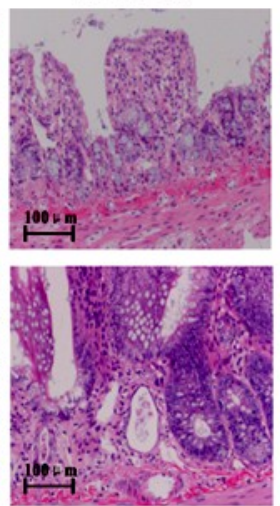

DXM
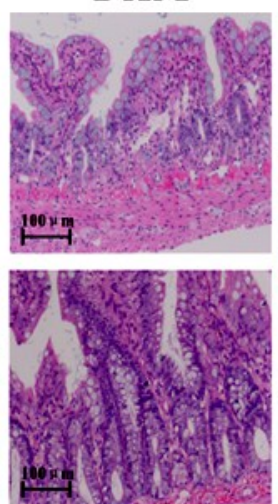

LGDI
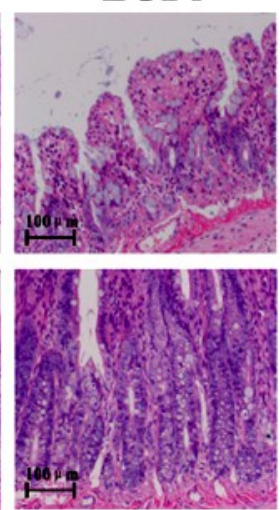

LGDh

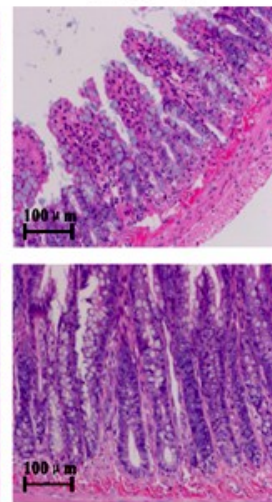

B

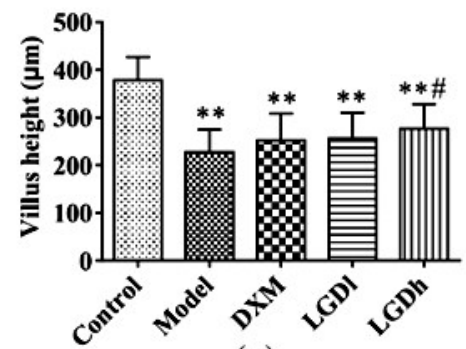

(a)

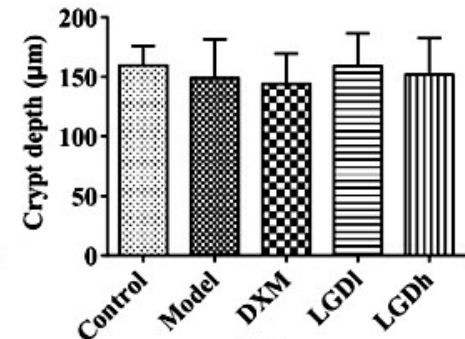

(b)

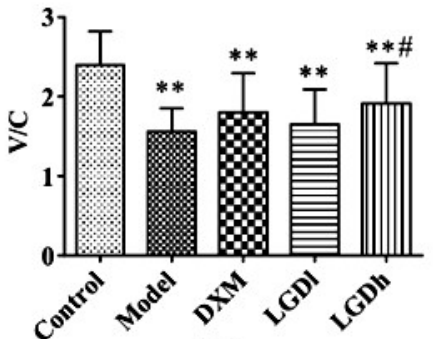

(c)

\section{C}

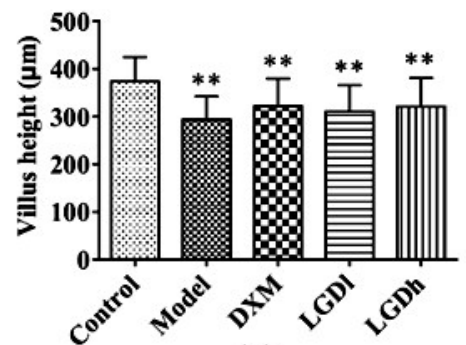

(a)

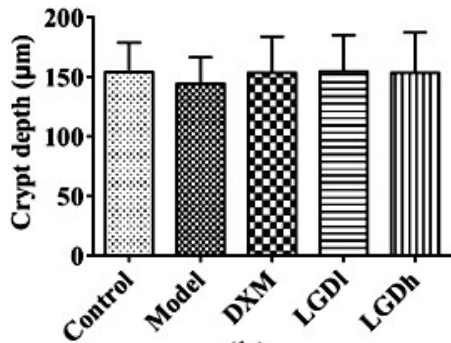

(b)

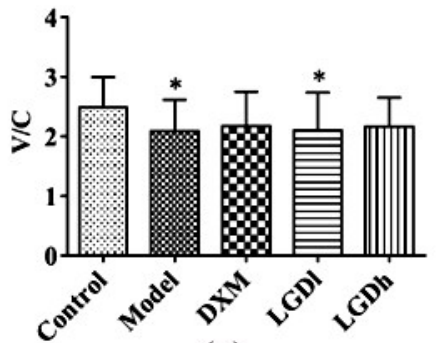

(c)

Figure 3. Effects of LGD on ileum histopathological changes. A: lleum histopathological changes on day 3 and 7. B: (a) Villus length, (b) crypt depth, and (c) V/C ratio on day 3. C: (a) Villus length, (b) crypt depth, and (c) V/C ratio on day 7 . Data are means \pm $\mathrm{SD} ;{ }^{*} \mathrm{P}<0.05$ and ${ }^{* *} \mathrm{P}<0.01$ versus control; ${ }^{\#} \mathrm{P}<0.05$ and ${ }^{\# \#} \mathrm{P}<0.01$ versus model. 
Wang et al. / Liangxue Guyuan decoction alleviates radiation enteritis

A Control

Model

DXM

LGDI

LGDh

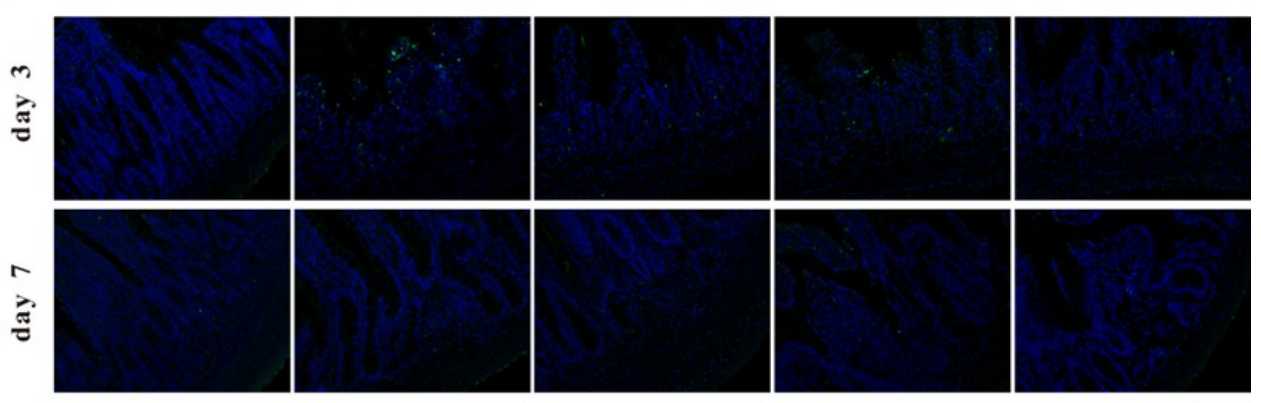

B

(a)
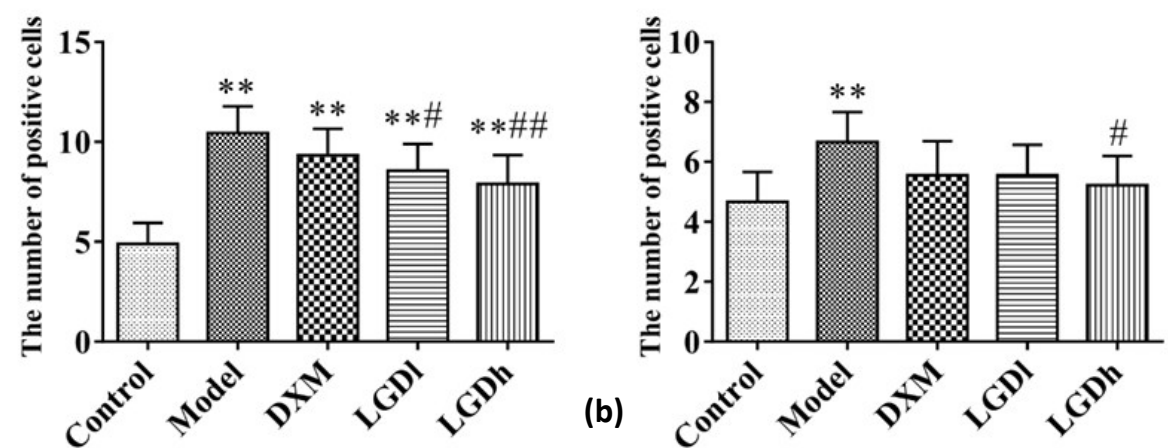

Figure 4. Effects of LGD on intestinal cell apoptosis on day 3 and 7. A: TUNEL expression (green) in the ileum on day 3. B: TUNEL expression in the ileum on day 7. C: TUNEL-positive cells comparison (a) on day 3 and (b) on day 7. Data are means \pm SD; ${ }^{*} P<0.05$ and ${ }^{* *} \mathrm{P}<0.01$ versus control; ${ }^{\#} \mathrm{P}<0.05$ and ${ }^{\# \#} \mathrm{P}<0.01$ versus model.

A
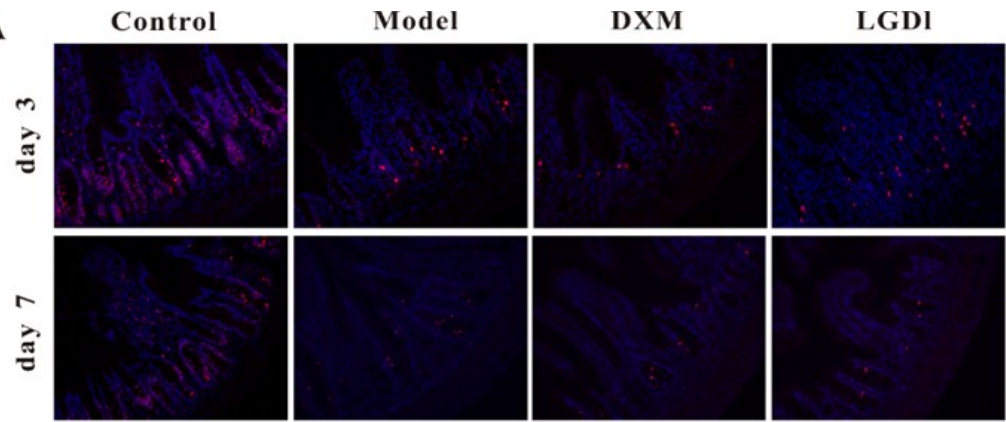

LGDh

B

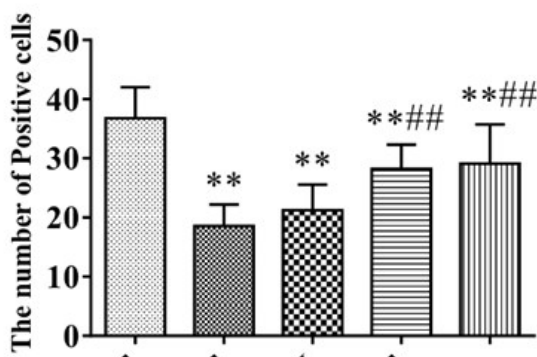

(a)

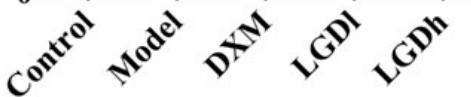

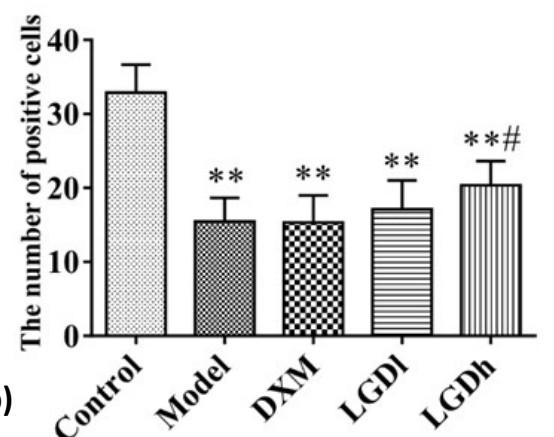

(b)

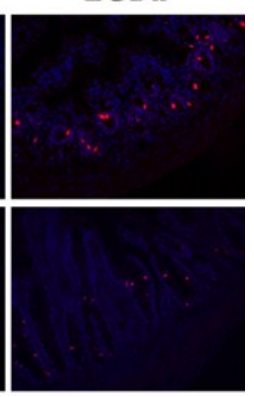

\section{列}




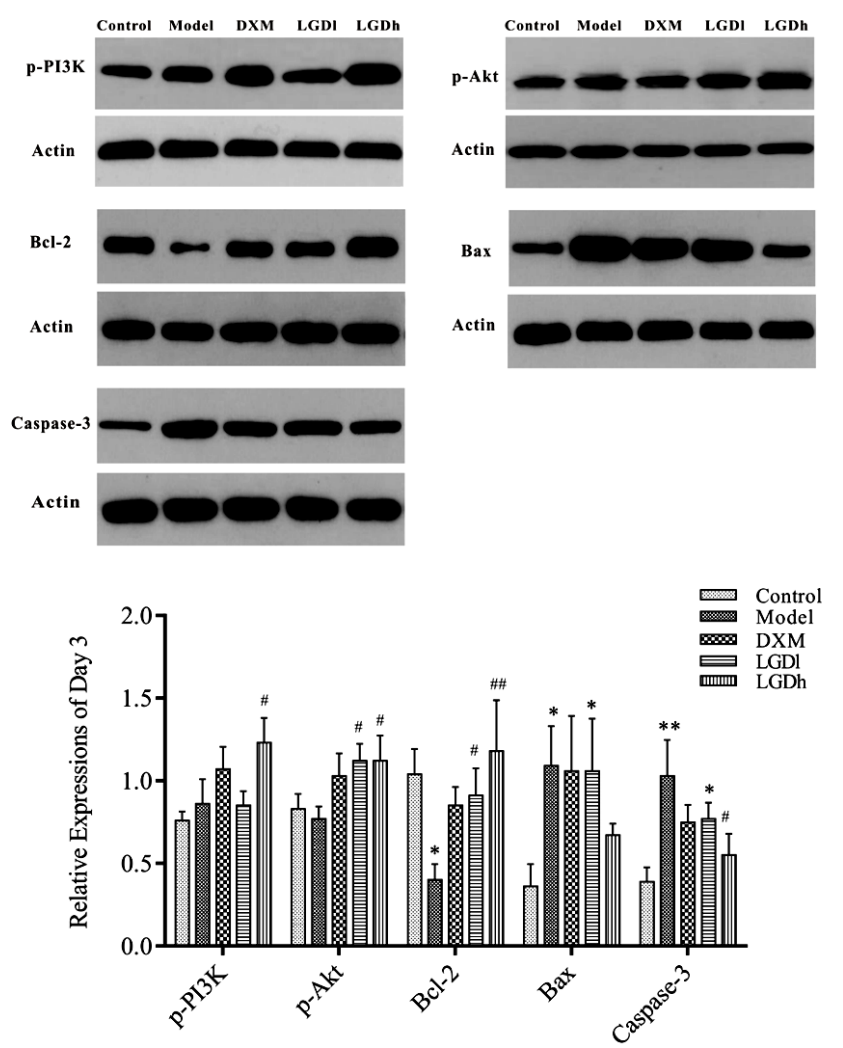

Figure 6. Effects of LGD on the expression of PI3K/AKT pathway-related proteins on day 3. Data are means $\pm \mathrm{SD}$;

${ }^{*} \mathrm{P}<0.05$ and ${ }^{* *} \mathrm{P}<0.01$ versus control; ${ }^{\#} \mathrm{P}<0.05$ and ${ }^{\# \#} \mathrm{P}<0.01$ versus model.

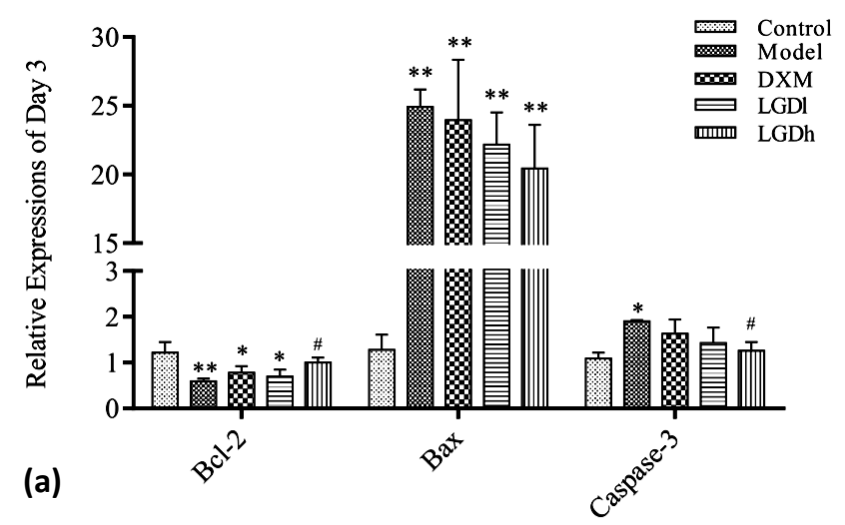

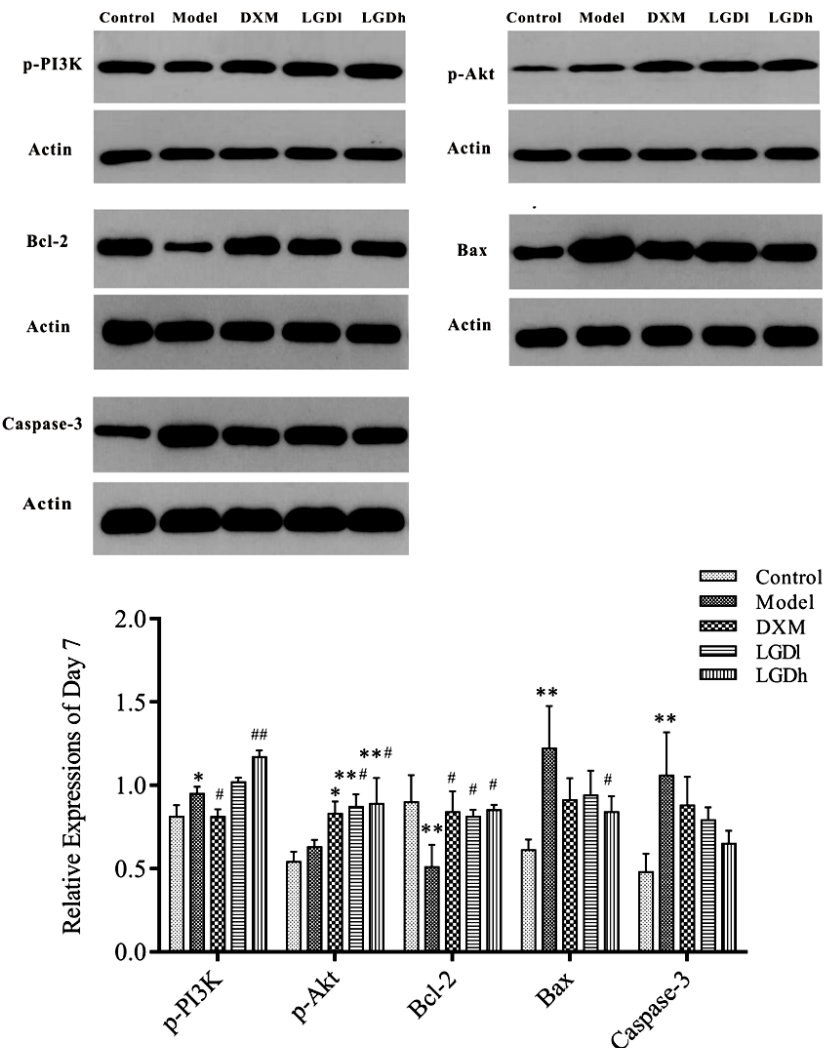

Figure 7. Effects of LGD on the expression of PI3K/AKT pathway-related proteins on day 7. Data are means $\pm S D$; ${ }^{*} \mathrm{P}<0.05$ and ${ }^{* *} \mathrm{P}<0.01$ versus control; ${ }^{\#} \mathrm{P}<0.05$ and ${ }^{\# \#} \mathrm{P}<0.01$ versus model.

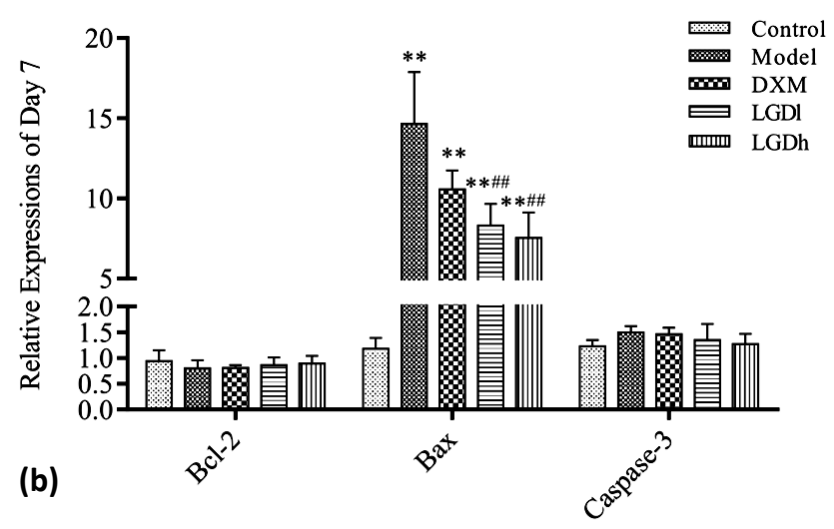

Figure 8. Effects of LGD on the mRNA expression of apoptosis-related factors on day 3 and 7. (a) Bcl-2, Bax and Caspase-3 mRNA levels on day 3. (b) Bcl-2, Bax and Caspase-3 mRNA levels on day 7. Data are means $\pm S D ;{ }^{*} \mathrm{P}<0.05$ and ${ }^{* *} \mathrm{P}<0.01$ versus control; ${ }^{\#} \mathrm{P}<0.05$ and ${ }^{\# \#} \mathrm{P}<0.01$ versus model.

\section{DISCUSSION}

Exposure to ionizing radiation can lead to damage of DNA, proteins, lipids, and other biological macromolecules (13). Although epithelial regeneration in the small intestine can alleviate tissue damage quickly, the pathological 786 changes caused by high-dose irradiation, especially in severe cases, often destroy the biological barrier owing to severe damage to the intestinal mucosa (14). The loss of the barrier results in a large amount of fluid loss and the entry of toxic substances from the intestine into the blood, resulting in severe water and

Int. J. Radiat. Res., Vol. 19 No. 4, October 2021 
electrolyte imbalance and sepsis, which are the main causes of treatment failure in patients with severe RE (15). Recent studies have shown that traditional Chinese medicine, natural extracts, and synthetic compounds can inhibit intestinal cell apoptosis, promote proliferation, reduce intestinal radiation damage, and improve survival time (16-19). Therefore, in this study, we observed the effects of LGD on survival time, intestinal pathology, and TUNEL and Ki67 expression in the ileum.

As shown in figures 2-5, the intestinal epithelial structure was destroyed, and the number of apoptotic cells and proliferating cells increased greatly after exposure to radiation in rats. However, with the proliferation and repair of intestinal epithelial cells, the number of apoptotic cells gradually reduced, and the intestinal epithelial cells were replenished. In this process, rats with poor resilience and poor drug response died. The LGDh group showed that a high dose of LGD extended the survival time of irradiated rats, promoted intestinal villus and crypt repair, decreased the number of TUNEL-positive cells, and increased the number of Ki67-positive cells. Therefore, we believe that LGD may prolong the survival time of rats by reducing the apoptosis of intestinal cells, promoting the recovery of the intestinal barrier, and reducing the degree of radiation-induced injury. This effect is better than that of dexamethasone.

To clarify the mechanism of LGD, we studied the expression of the PI3K/AKT pathway. Studies have shown that radiation can activate PI3K and produce phosphatidylinositol-3phosphate to expose the phosphorylation sites of AKT. With the catalysis of 3-phosphoinositidedependent protein kinase 1 (PDK1), AKT is phosphorylated and then transferred to the cells. Subsequently, Bcl-2 was released to play an anti-apoptotic role. Activated AKT can also directly inhibit the activities of apoptotic factors Bax and caspase- 3 (20). Caspase-3, a death protease, is a mediator and executor of the apoptotic pathway and is the most important protease involved in the execution of apoptotic cells (21). Activated caspase- 3 can block the cell cycle by cleaving DNA-dependent protein kinase and polyadenosine diphosphate ribose polymerase, destroying the cell repair mechanism, which leads to cell disintegration and eventually death (22-24). Bcl-2 and Bax are the most important factors in cell apoptosis. Bax can be translocated to the mitochondrial membrane by the stimulus of the death signal to form channels, release cytochrome $\mathrm{c}$ and other pro-apoptotic factors, activate caspase-3, and initiate the caspase cascade reaction (25-26). Bcl-2 can form a heterodimer with Bax, inhibit the translocation and dimerization of Bax, and thereby inhibit apoptosis (27-28). In addition, Bcl-2 and Bax can also act as substrates for caspase-3 and are interrelated and mutually restrictive in apoptotic transmission (22).

On day 3, in the LGDh group, the expressions of p-PI3K, p-AKT, and Bcl-2 had increased and the expression of caspase- 3 was reduced at the protein and mRNA levels, indicating that LGDh could inhibit apoptosis and promote proliferation by increasing the expression of p-AKT and Bcl-2 and inhibiting the expression of caspase-3. On day 7, although intestinal injury was gradually repaired, the phenomenon of apoptosis and proliferation of intestinal cells still existed. The expressions of p-PI3K, p-AKT, and Bcl-2 in the LGDh group had increased, while Bax expression at protein and gene levels decreased. These results suggested that on day 7, LGDh still improved cell survival by regulating the expression of PI3K/AKT pathway-related factors. Therefore, we believe that a high dose of LGD can inhibit apoptosis, promote mucosal repair, alleviate radiation damage, and increase the survival rate of rats by upregulating the PI3K/AKT pathway.

The treatment of $\mathrm{RE}$ is challenging, and Chinese medicine is an important resource in the treatment of many diseases, including SARS (29), Ebola (30), and Malaria (31). For example, Tu Youyou was a co-recipient of the 2015 Nobel Prize in Medicine for her discovery of the antimalarial drug artemisinin, extracted from Artemisia Annua, a herb used in Chinese medicine. Therefore, we examined the efficacy of LGD and explored the mechanism of its action. Our results demonstrated that LGD prolonged the survival time of irradiated rats, inhibited 
intestinal epithelial cell apoptosis, and promoted intestinal repair.

The mechanism of action of LGD may be related to the regulation of caspase- 3 , Bax, and Bcl-2. However, there are still many questions, such as which components of LGD play a major role, their underlying mechanism of action, and whether they have a regulatory effect on other signaling pathways related to apoptosis and proliferation. Therefore, we intend to conduct further research. First, we plan to use gas chromatography-mass spectrometry to identify the active components of LGD, such as ginsenoside $\mathrm{Rg} 1$, and then conduct in vitro experiments to determine the anti-apoptosis mechanism of RE. We hope that our results will provide new drugs for the treatment of RE.

\section{FUNDING}

This work was supported by Logistics Scientific Research Program (No: AWS14C014)

\section{ACKNOWLEDGEMENTS}

We sincerely thank $\mathrm{Dr}$. Hangchen $\mathrm{Xu}$ for helping us with the experiment and analyzing the data. We also thank Professor Yue Gao and Professor Liang Yang for valuable advice.

\section{Conflicts of interest: Declared none.}

\section{REFERENCES}

1. Gudkov SV, Guryev EL, Gapeyev AB, Sharapov MG, Bunkin NF, Shkirin AV, et al. (2019) Unmodified hydrated C 60 fullerene molecules exhibit antioxidant properties, prevent damage to DNA and proteins induced by reactive oxygen species and protect mice against injuries caused by radiation-induced oxidative stress. Nanomedicine, 15: 37-46.

2. Jalili-Firoozinezhad S, Prantl-Baun R, Jiang A, Potla $\mathrm{R}$, Mammoto T, Weaver JC, et al. (2018) Modeling radiation injury-induced cell death and countermeasure drug responses in a human Gut-on-a-Chip. Cell Death \& Disease, 9: 223-237.

3. Pejchal J, Sinkorova Z, Tichy A, Pruchova S, Kmochova A, Durisova A, et al. (2016) Epidermal Growth Factor Attenuates Delayed lonizing Radiation-Induced Tissue Damage in
Bone Marrow Transplanted Mice. Radiation Research, 186: 264-274.

4. Jing Z, Lian BL, Zhu Q, Ren HB, Wu JY, Wang T, et al. (2015) Intravenous injections of human mesenchymal stromal cells modulated the redox state in a rat model of radiation myelopathy. Evidbased Compl Alt, 23: 432369.

5. Posadas SJ, Largo C, Merino JJ, Elvira M, Gonzalez G, Caz $V$, et al. (2011) Growth hormone upregulates intestinal trefoil factor expression in the ileum of rats after $\gamma$ radiation. Exp Biol Med, 236: 205-211.

6. Yao Y, Zheng Z, Song Q (2018) Mesenchymal stem cells: A double-edged sword in radiation-induced lung injury. Thoracic Cancer, 9: 208-217.

7. SJ Bing, MJ Kim, G Ahn, J Im, DS Kim, D Ha, et al. (2014) Acidic polysaccharide of Panax ginseng regulates the mitochondria/caspase-dependent apoptotic pathway in radiation-induced damage to the jejunum in mice. Acta Histochemica, 116: 514-521.

8. $H$ Dan, L Zhang, $X$ Qin, $X$ Peng, $M$ Wong, $X$ Tan, et al. (2016) Moutan cortex extract exerts protective effects in a rat model of cardiac ischemia/reperfusion. Can J Physiol Pharm, 94: 245-250.

9. Z Yu, Y Wang, W Lei, Y Zhang, Y Qin, T Chen, et al. (2012) Effects of Rehmannia glutinosa oligosaccharide on human adipose-derived mesenchymal stem cells in vitro. Life Sci, 91: 25-26.

10. Z Ke, G Wang, L Yang, H Qiu, H Wu, M Du, et al. (2017) Crude terpene glycoside component from Radix paeoniae rubra protects against isoproterenol-induced myocardial ischemic injury via activation of the PI3K/AKT/mTOR signaling pathway. J ethnopharmacology, 206: 160-169.

11. Liu R, Wang M, Duan JA, Guo JM, Tang YP (2010) Purification and identification of three novel antioxidant peptides from Cornu Bubali (water buffalo horn). Peptdes, 31: 786793.

12. MM Mutsumi, S Kazuko, O Kumio, N Toshiyuki, N Masahiro, U Takashi, et al. (2008) Protection by polaprezinc against radiation-induced apoptosis in rat jejunal crypt cells. J Radiat Res, 49: 341-357.

13. Ma J, Marignier JL, Pernot P (2018) Direct observation of the oxidation of DNA bases by phosphate radicals formed under radiation: a model of the backbone-to-base hole transfer. Phys Chem Chem Phys, 20: 14927-14937.

14. Riehl T, Cohn S, Tessner T, Schloemann S, Stenson WF (2000) Lipopolysaccharide is radioprotective in the mouse intestine through a prostaglandin-mediated mechanism. Gastroenterology, 118:1106-1116.

15. H Joyeux, J Matias, MC Gouttebel, JB Vedrenne, B SaintAubert (1994) Therapeutic strategy in 46 cases of radiation injury of the intestine. Chirurgie, 120: 129-133.

16. Wang X, Wei L, Cramer JM, Leibowitz BJ, Judge C, Epperly $M$, et al. (2015) Pharmacologically blocking p53-dependent apoptosis protects intestinal stem cells and mice from radiation. Sci Rep, 5: 8566.

17. Qiu W, Leibowitz B, Zhang L, Yu J (2010) Growth factors protect intestinal stem cells from radiation-induced apoptosis by suppressing PUMA through the PI3K/AKT/p53 axis. 
Oncogene, 29: 1622-1632.

18. BK Park, HL Ju, HW Seo, KS Oh, BH Lee (2019) Icariin protects against radiation-induced mortality and damage in vitro and in vivo. Int J of Radiat Biol, 4: 1-9.

19. Lambros MP, Kondapalli L, Parsa C, Mulamalla HC, Orlando $\mathrm{R}$, Pon D, et al. (2015) Molecular signatures in the prevention of radiation damage by the synergistic effect of $\mathrm{N}$ acetyl cysteine and qingre liyan decoction, a traditional chinese medicine, using a 3-dimensional cell culture model of oral mucositis. Evidbased Compl Alt, 2015: 425760.

20. Pan S, Sun Y, Sui D, Yang T, Fu S, Wang J, et al. (2018) Lobaplatin promotes radiosensitivity, induces apoptosis, attenuates cancer stemness and inhibits proliferation through PI3K/AKT pathway in esophageal squamous cell carcinoma. Biomed \& Pharmacother, 2018: 567-574.

21. Odonkor CA and Achilefu S (2009) Modulation of effector caspase cleavage determines response of breast and lung tumor cell lines to chemotherapy. Cancer Invest, 27: 417429.

22. W Guo, Y Zhang, Z Ling, X Liu, X Zhao, Z Yuan, et al. (2015) Caspase-3 feedback loop enhances Bid-induced AIF/endoG and Bak activation in Bax and p53-independent manner. Cell Death Dis, 6:e1919.

23. Y Yosefzon, D Soteriou, A Feldman, L Kostic, E Koren, S Brown, et al. (2018) Caspase-3 Regulates YAP-Dependent Cell Proliferaton and Organ Size. Mol Cell, 70: 573-587.

24. Deshpande RP and Babu PP (2018) pDok2, caspase 3 de- pendent glioma cell growth arrest by nitidine chloride. Pharmacol Rep, 70: 48-54.

25. Scorrano L and Korsmeyer SJ (2003) Mechanisms of cytochrome $\mathrm{c}$ release by proapoptotic $\mathrm{BCL}-2$ family members. Biochem Bioph Res Com, 304: 437-444.

26. Jiang W, Chen Y, Li B, Gao SY (2017) DBA-induced caspase3-dependent apoptosis occurs through mitochondrial translocation of cyt-c in the rat hippocampus. Mol BioSyst, 13:1863-1873.

27. Renault TT, Dejean LM, Manon S (2017) A brewing understanding of the regulation of Bax function by $\mathrm{Bcl}-\mathrm{xL}$ and $\mathrm{BCl}$ -2. Mech Ageing and Dev, 161: 201-210.

28. Kvansakul M and Hinds MG (2015) The Bcl-2 family: structures, interactions and targets for drug discovery. Apoptosis, 20: 136-150.

29. Liu X, Zhang M, He L, Li Y (2012) Chinese herbs combined with Western medicine for severe acute respiratory syndrome (SARS). Cochrane Db Syst Rev, 10: D4882.

30. Wang Z, Liang $\mathrm{H}$, Cao $\mathrm{H}$, Zhang B, Li J, Wang W, et al. (2019) Efficient ligand discovery from natural herbs by integrating virtual screening, affinity mass spectrometry and targeted metabolomics. Analyst, 144: 2881-2890.

31. Li J, Zhang C, Gong M, et al. (2018) Combination of artemisinin-based natural compounds from Artemisia annual for the treatment of malaria: Pharmacodynamic and pharmacokinetic studies. Phytother Res, 32: 1415-1420. 
